\section{Smith ScholarWorks}

$1-1-2014$

\section{Nanochromosome Copy Number Does Not Correlate with RNA Levels Though Patterns Are Conserved Between Strains of the Ciliate Morphospecies Chilodonella uncinata}

Jie Huang

Smith College

Laura A. Katz

Smith College, Ikatz@smith.edu

Follow this and additional works at: https://scholarworks.smith.edu/bio_facpubs

Part of the Biology Commons

\section{Recommended Citation}

Huang, Jie and Katz, Laura A., "Nanochromosome Copy Number Does Not Correlate with RNA Levels Though Patterns Are Conserved Between Strains of the Ciliate Morphospecies Chilodonella uncinata" (2014). Biological Sciences: Faculty Publications, Smith College, Northampton, MA.

https://scholarworks.smith.edu/bio_facpubs/107

This Article has been accepted for inclusion in Biological Sciences: Faculty Publications by an authorized administrator of Smith ScholarWorks. For more information, please contact scholarworks@smith.edu 


\title{
Nanochromosome Copy Number Does not Correlate with RNA Levels Though Patterns are Conserved between Strains of the Ciliate Morphospecies Chilodonella uncinata
}

\author{
Jie Huang ${ }^{\mathrm{a}, \mathrm{b}}$ and Laura A. Katz ${ }^{\mathrm{a}, \mathrm{c}, 1}$ \\ aDepartment of Biological Sciences, Smith College, Northampton, MA 01063, USA \\ ${ }^{b}$ Key Laboratory of Aquatic Biodiversity and Conservation of Chinese Academy of Sciences, \\ Institute of Hydrobiology, Chinese Academy of Sciences, Wuhan 430072, China \\ 'Program in Organismic and Evolutionary Biology, UMass-Amherst, Amherst, MA 01003, USA
}

\begin{abstract}
In some ciliates, extensive genome fragmentation leads to a macronucleus (i.e. somatic nucleus) containing gene-sized chromosomes that vary in copy number. Yet the relationship between copy number and expression level is not well understood as previous work has shown a variety of patterns. For example, nanochromosome copy numbers are positively correlated to mRNA levels in spirotrichous ciliates, while one study of Chilodonella uncinata suggested that they were inversely correlated. To study further copy number and expression levels in $C$. uncinata, we analyzed 11 members in five gene families (SSU-rDNA, actin, alpha-tubulin, histidine acid phosphatase family protein and protein kinase domain containing protein) from one strain. We find that macronuclear copy numbers of these genes range from hundreds to thousands per cell, and that copy number does not correlate with expression level as measured by steady-state RNA in predominantly-vegetative cultures. We also compared six of these genes to their orthologs in a second genetically-isolated strain to reveal that patterns of nanochromosome and transcript copy numbers are conserved between strains. Our data suggest that nanochromosome copy number may be related to a feature like nuclear architecture.
\end{abstract}

\section{Keywords}

Chilodonella; ciliate; copy number variation; RNA levels; quantitative PCR

\section{Introduction}

Ciliates are single-cell eukaryotes characterized by the presence of hair-like cilia and two types of nuclei. The small diploid micronucleus (MIC) serves as the germline nucleus,

\footnotetext{
(c) 2014 Elsevier GmbH. All rights reserved.

${ }^{1}$ Corresponding author; fax +1 413-585-3786, 1katz@ smith.edu (L.A. Katz).
}

Publisher's Disclaimer: This is a PDF file of an unedited manuscript that has been accepted for publication. As a service to our customers we are providing this early version of the manuscript. The manuscript will undergo copyediting, typesetting, and review of the resulting proof before it is published in its final citable form. Please note that during the production process errors may be discovered which could affect the content, and all legal disclaimers that apply to the journal pertain. 
which is transcriptionally inactive, whereas transcription occurs in the somatic macronucleus (MAC). After sexual conjugation, the old MAC is degraded and a new MAC develops from a zygotic nucleus. During the MAC development, the zygotic genome undergoes massive rearrangements including fragmentation of chromosomes, DNA elimination, and amplification (e.g. Prescott 1994). In three classes of ciliates (Spirotrichea, Armophorea and Phyllopharyngea), DNA was extensively fragmented, creating extremely short chromosomes that typically contain single genes (e.g. Riley and Katz 2001).

Previous studies of varying species of ciliates showed different patterns between macronuclear copy number and expression levels. A positive correlation between copy number and gene expression for three pheromone genes exists in Euplotes raikovi (CI: Spirotrichea, La Terza et al. 1995). In Oxytricha trifallax (CI: Spirotrichea), the copy numbers of $11 \mathrm{MAC}$ chromosomes are moderately correlated to gene expression levels, though the pattern is not conserved in closely related species or even between two subpopulations of one strain (Xu et al. 2012). In contrast, a recent study in Chilodonella uncinata revealed that members of the $\beta$-tubulin gene family that are abundant in the macronucleus tend to be expressed at low levels, while rarer gene family members are expressed at higher levels (Bellec and Katz 2012).

In this study, we use quantitative real-time PCR (qPCR) to assess further the copy number variation and RNA levels in cultures of predominantly-vegetative cells of $C$. uncinata. Protein kinase domain containing protein $(P k c)$ and histidine acid phosphatase family protein (Hap) are two alternatively processed gene families characterized from analyzing the transcriptome data of $C$. uncinata (Gao et al. submitted). We analyzed 8 members of these two gene families (3 Hap family members, 5 P $k$ family members) from the POL strain of C. uncinata. We also compared copy numbers of three essential genes (SSU-rDNA, actin and alpha-tubulin) and the three members of Hap gene family in the Pol strain to that in the USA-SC2 strain of $C$. uncinata.

\section{Results}

\section{Copy Numbers and RNA Levels of 11 Genes per Cell in the POL Strain of C. uncinata (Fig. 1A)}

We estimated the macronuclear copy numbers and RNA levels of three essential genes (SSU-rDNA, actin and a-tubulin), three Hap (histidine acid phosphatase family protein) gene family members and five $P k c$ (protein kinase domain containing protein) gene family members in the POL strain of $C$. uncinata (Fig. 1A). Macronuclear copy numbers of the 11 genes differ from one another, ranging from a few hundreds to several thousands of copies per cell (Fig. 1A, Table 1). However, unlike in spirotrichous ciliates (La Terza et al. 1995; $\mathrm{Xu}$ et al. 2012), our data do not show a clear correlation between gene expression levels and their abundances in the macronucleus during vegetative growth of $C$. uncinata as some abundant chromosomes are rarely expressed (e.g. Hap and $P k c$ ) while others like actin are more highly expressed (Fig. 1A). Given that $C$. uncinata independently evolved gene-sized macronuclear chromosomes from spirotrichous ciliates (Riley and Katz 2001), it is not surprising if different mechanisms of regulating gene expression are found in C. uncinata and other ciliates with heavily fragmented macronuclear chromosomes. 
SSU is the most abundant chromosome, with $5.92 \times 10^{4}$ copies per cell. During vegetative growth, the copy number of ribosomal RNA per cell $\left(5.58 \times 10^{5}\right.$ copies $)$ is roughly 10 fold over the macronuclear rDNA copy numbers (Table 1). Alpha-tubulin is the second most abundant gene among the 11 genes we quantified, and its copy number is nearly 5 times that of actin $\left(8.5 \times 10^{3}\right.$ vs. $1.82 \times 10^{3}$ copies per cell, Table 1 ; Fig. 1A). These two essential genes are expressed at similar levels $\left(1.26 \times 10^{3}\right.$ vs. $1.62 \times 10^{3}$ copies per cell $)$ despite their 5 fold difference in copy number (Table 1, Fig. 1A).

Among the three Hap gene family members, Hap-P2 has the highest copy number per cell $\left(1.7 \times 10^{3}\right.$ copies $)$, followed by that of Hap-P1 $\left(1.19 \times 10^{3}\right.$ copies $)$ and Hap-P3 $\left(0.65 \times 10^{3}\right.$ copies; Fig. 1A). In the $P k c$ gene family, the copy numbers range from seven hundred ( $P k c$ $\mathrm{P} 2$ and $P k c-\mathrm{P} 3)$ to nearly three thousand ( $P k c$-P4) copies per cell (Fig. 1A). In contrast, all the gene family members for both $P k c$ and Hap have quite low expression, with fewer than 10 transcripts per cell (Fig. 1A).

\section{Copy Numbers and RNA Levels of 6 Genes in the USA-SC2 Strain of $C$. uncinata}

To compare patterns of copy numbers and expression levels between strains, we also quantified orthologs of six genes from the USA-SC2 strain of $C$. uncinata. Both strains showed similar patterns of nanochromosome copy numbers and RNA levels. For example, the macronuclear copy number of alpha-tubulin is about 5 times that of actin while these two essential genes are expressed at similar levels in both strains (Fig. 1 and Table 1). The one exception is the mRNA level of Hap-P3 where we observed different expression between strains; in the POL strain, Hap-P3 was expressed at the highest level among the three Hap gene family members while it had lower expression than Hap-P2 in the USA-SC2 strain (Fig. 1).

\section{Discussion}

In this study, we observed that macronuclear copy numbers of the 11 genes varied from a few hundred (Hap-P3) to tens of thousands (SSU-rDNA) copies per cell in the POL strain of C. uncinata, and that macronuclear copy numbers are not correlated with RNA levels (Table 1; Figure 1). The SSU-rDNA has much higher copy numbers than other genes, which is consistent with all previous work in other ciliates (e.g. Prescott 1994). The variable copy numbers is similar to data from Euplotes crassus (Spirotrichea; Baird and Klobutcher 1991) indicating that copy number is regulated individually for each macronuclear chromosome in these two ciliates. Unlike in spirotrichous ciliates (La Terza et al. 1995; Xu et al. 2012), RNA levels in predominantly vegetative cultures of $C$. uncinata are not positively correlated to gene copy numbers in the macronucleus; instead steady-state mRNA levels appear uncorrelated as high macronuclear copy numbers sometimes associate with high expression (e.g. actin, alpha-tubulin) and sometimes very low expression (e.g. Pkc and Hap, Fig. 1).

We recognize that there are caveats in our estimates. For example, the proxy for expression levels used this study is RNA levels in non-synchronous cultures, which are effected not only by transcription rate but also by RNA stability. In addition, the extra manipulations in generating cDNAs as compared to isolate DNAs (e.g. efficiency of extraction and reverse transcription) may affect the absolute numbers in estimates of expression levels. Similarly, 
our cultures are not synchronized but instead tend to have $~ 90-95 \%$ of cells in the vegetative phases (e.g. neither actively dividing nor in conjugation). However, this lack of synchrony would only impact copy number by a factor of two as cells transition between mitosis and $\mathrm{S}$ phases.

The lack of correlation between macronuclear copy number and expression levels in $C$. uncinata suggests several alternative explanations. For example, macronuclear copy number may be related to nuclear architecture given the heteromeric nature of the macronucleus of C. uncinata - the macronucleus contains both DNA rich and DNA poor regions (Radzikowski 1985; Raikov 1996). Under this model, lowly expressed macronuclear chromosomes (e.g. Hap and $P k c$ ) may be located in the dense chromatin granules of the DNA rich region (Bellec et al. 2014; Radzikowski 1985), where transcription requires unwinding of heterochromatin. Alternatively, the low expression of chromosomes may be related to their origins as the genes with the lowest expressions here, Pkc and Hap, are generated by alternative processing during macronuclear development (i.e. are generated using a combination of unique and shared micronuclear regions, Gao et al. 2014). Two of the three rarely expressed $\beta$-tubulin members (Bellec and Katz 2012) are also generated from alternatively processing of micronuclear loci (Katz and Kovner 2010), suggesting that alternatively processed gene family members may be expressed at low levels during vegetative growth of $C$. uncinata. Finally, the low expression of members of Hap and Pkc may indicate that they are cell-cycle dependent and repressed during vegetative growth, as has been seen for gene family members in other ciliates. For example, the transcription of one $\gamma$-tubulin gene in Euplotes focardii is dependent on the cell cycle stages (Marziale et al. 2008) while Stylonychia lemnae have a short-termed boost of differential expression of numerous genes during conjugation (Paschka et al. 2005).

In contrast to the lack of correlation between copy number and expression levels, patterns of macronuclear chromosome copy number and expression levels are conserved between two genetically isolated strains of $C$. uncinata. We observed similar patterns of copy numbers of three essential genes (SSU, actin and a-tubulin) and three Hap gene family members between two strains of $C$. uncinata (Table 1; Figure 1). In addition, the pattern of three essential gene copy numbers are stable compared to that quantified two years ago (Bellec and Katz 2012), which corresponds with the recent report that gene copy numbers in $C$. uncinata do not change under a stochastic process during vegetative growth (Spring et al. 2013). This is different from spirotrichous ciliates where copy number patterns are not conserved in closely related species or even between two subpopulations of one strain (Xu et al. 2012). However, during sexual reproduction, there is evidence showing that parental RNA can epigenetically regulate the copy number variation of genes within strains of Stylonychia and Oxytricha (Heyse et al. 2010; Nowacki et al. 2010).

\section{Synthesis}

Generally, regulation of transcription of gene family members in eukaryotes is a result of the combined effects of copy numbers of paralogous genes and the interactions of transcription factors (Remenyi et al. 2004; Sproul et al. 2005; Struhl 1999). In contrast, the highly polyploid macronuclear chromosomes of ciliates like $C$. uncinata provide the opportunity 
for regulating gene expression by changing gene copy numbers by differentially amplifying individual macronuclear chromosomes. However, we find no simple correlation as genes on chromosomes with similarly high copy numbers are expressed at varying levels (Table 1; Fig. 1). Instead, we find that both copy number and expression levels are similar for orthologs compared between strains. Given that expression levels are determined not only by transcription rates but also by degradation rates, and mRNAs have a dynamic range of half-lives (Molin et al. 2009), it's not surprising that gene expression is decoupled from copy number of chromosomes in $C$. uncinata.

\section{Methods}

\section{Cell line and cell culture}

The two strains of $C$. uncinata $(\mathrm{POL}=\mathrm{ATCC} \circledast \mathrm{PRA}-256$, USA-SC2) used in this study were described previously (Katz et al. 2011; Robinson and Katz 2007) and culturing conditions are as reported in these publications.

\section{DNA and RNA extraction}

Cultures were filtered through a $10 \mu \mathrm{m}$ filter and starved for two days, then pelleted by centrifugation at $2500 \mathrm{x} g$. Numbers of cells were estimated by averaging three counts of 1 $\mu \mathrm{l}$ from the pellet. Total genomic DNA from pelleted cells was extracted using a phenol/ chloroform protocol (Ausubel et al. 1993). RNA was extracted from cell pellets using the Qiagen RNeasy Mini kit (cat. \# 74104) and quantified using Qubit ${ }^{\mathrm{TM}}$ RNA Assay Kit (cat. \# Q32852, Invitrogen, CA) on Qubit 2.0 Fluorometer. TURBO DNA-free ${ }^{\mathrm{TM}}$ Kit (cat. \# AM1907, Ambion, CA) was used to remove contaminating DNA. DNase treated RNA was then subjected to cDNA synthesis using the QuantiTect Rev. Transcription kit (Qiagen, cat. \# 205311) according to the manufacturer's instructions. Random primers were used to prime the first strand cDNA syntheses.

\section{Quantitative PCR}

Gene copy numbers and their expression level were both assessed by using absolute quantitative real-time PCR (qPCR). Standard curves built on cycle thresholds (Ct) derived from ten-fold serially diluted plasmids ( $1 \mathrm{ng}$ to $10^{-7} \mathrm{ng}$ ) containing gene fragments of interest were used to calculate copy numbers of genes and their cDNA. To obtain the copy numbers per cell, absolute gene copy numbers were interpolated from standard curves and divided by the estimated number of cells. Primers were designed on the non-conserved regions of genes (Genbank accession numbers see Table 2), and checked the selfcomplementarity using Oligo Calc program (Kibbe 2007). Two sets of primers were used to assess consistency in copy numbers calculated from qPCR. Quantitative PCR was carried out using DyNAmo Flash SYBR Green qPCR kit (Fisher Scientific, USA) in 96-well plates on an ABI StepOnePlus thermal-cycler. Reactions were conducted in a final volume of 20 $\mu \mathrm{L}$, containing $10 \mu \mathrm{L} 2 \times$ master mix, $150 \mathrm{nM}$ of each primer, $1 \mu \mathrm{L}$ of template DNA, and 8 $\mu \mathrm{L}$ of water. Cycling conditions were: $95^{\circ} \mathrm{C}$ for $10 \mathrm{~min}$ followed by 40 cycles of $95^{\circ} \mathrm{C}$ for $15 \mathrm{~s}$ and $60^{\circ} \mathrm{C}$ for $1 \mathrm{~min}$, and a melting curve stage $\left(95^{\circ} \mathrm{C} 15 \mathrm{~s}, 60^{\circ} \mathrm{C} 30 \mathrm{~s}\right.$, and $95^{\circ} \mathrm{C} 15 \mathrm{~s}$ ) was added to ensure PCR specificity. Three and two individual qPCR experiments (in triplicates) were performed for DNA and cDNA, respectively. 


\section{Acknowledgments}

We thank Eric Meyer and two anonymous reviewers for their comments and suggestions. We would also like to thank Feng Gao (Ocean university of China) for sharing the macronuclear sequences of genes of Pkc and Hap. Thanks also to Jessica R. Grant and Lou Ann Bierwert (Smith College) for technical help. This work is supported by an AREA award from the National Institutes of Health (1R15GM097722) to L.A. Katz.

\section{References}

Ausubel, FM.; Brent, R.; Kingston, RE.; Moore, DD.; Seidman, JG.; Smith, JA.; Struhl, K. Current Protocols in Molecular Biology. Wiley-Liss; New York: 1993.

Baird SE, Klobutcher LA. Differential DNA amplification and copy number control in the hypotrichous ciliate Euplotes crassus. J Protozool. 1991; 38:136-140. [PubMed: 1902260]

Bellec L, Katz LA. Analyses of chromosome copy number and expression level of four genes in the ciliate Chilodonella uncinata reveal a complex pattern that suggests epigenetic regulation. Gene. 2012; 504:303-308. [PubMed: 22588027]

Bellec L, Maurer-Alcala XX, Katz LA. Characterization of the life cycle and heteromeric nature of the macronucleus of the ciliate Chilodonella uncinata using fluorescence microscopy. J Eukaryot Microbiol. 201410.1111/jeu.12109

Gao F, Song W, Katz LA. Genome structure drives patterns of gene family evolution in ciliates, a case study using Chilodonella uncinata (Protista, Ciliophora, Phyllopharyngea). Evolution. 2014 (in press).

Heyse G, Jönsson F, Chang WJ, Lipps HJ. RNA dependent control of gene amplification. Proc Natl Acad Sci USA. 2010; 107:22134-22139. [PubMed: 20974970]

Katz LA, Kovner AM. Alternative processing of scrambled genes generates protein diversity in the ciliate Chilodonella uncinata. J Exp Zool B Mol Dev Evol. 2010; 314B:480-488. [PubMed: 20700892]

Katz LA, DeBerardinis J, Hall MS, Kovner AM, Dunthorn M, Muse SV. Heterogeneous rates of molecular evolution among cryptic species of the ciliate morphospecies Chilodonella uncinata. $\mathrm{J}$ Mol Evol. 2011; 73:266-272. [PubMed: 22258433]

Kibbe WA. OligoCalc: an online oligonucleotide properties calculator. Nucleic Acids Res. 2007; 35:W43-W46. [PubMed: 17452344]

La Terza A, Miceli C, Luporini P. Differential amplification of pheromone genes of the ciliate Euplotes raikovi. Dev Genet. 1995; 17:272-279. [PubMed: 8565333]

Marziale F, Pucciarelli S, Ballarini P, Melki R, Uzun A, Ilyin VA, Detrich HW III, Miceli C. Different roles of two $\gamma$-tubulin isotypes in the cytoskeleton of the Antarctic ciliate Euplotes focardii. FEBS Journal. 2008; 275:5367-5382. [PubMed: 18959762]

Molin C, Jauhiainen A, Warringer J, Nerman O, Sunnerhagen P. mRNA stability changes precede changes in steady-state mRNA amounts during hyperosmotic stress. RNA. 2009; 15:600-614. [PubMed: 19223440]

Nowacki M, Haye JE, Fang W, Vijayan V, Landweber LF. RNA-mediated epigenetic regulation of DNA copy number. Proc Natl Acad Sci USA. 2010; 107:22140-22144. [PubMed: 21078984]

Paschka AG, Horejschi V, Jonsson F, Lindecke A, Weier G, Kaltschmidt C, Lipps HJ. A microarray analysis of developmentally regulated genes during macronuclear differentiation in the stichotrichous ciliate Stylonychia lemnae. Gene. 2005; 359:81-90. [PubMed: 16150557]

Prescott DM. The DNA of ciliated protozoa. Microbiol Rev. 1994; 58:233-267. [PubMed: 8078435]

Radzikowski S. Replication division and mechanisms controlling the variable DNA content in the heteromeric macronucleus of Chilodonella steini. Arch Protistenkd. 1985; 130:381-396.

Raikov, IB. Nuclei of Ciliates. In: Hausmann, K.; Bradbury, PC., editors. Ciliates: Cells as Organisms. Gustav Fischer; Stuttgart: 1996. p. 221-243.

Remenyi A, Scholer H, Wilmanns M. Combinatorial control of gene expression. Nat Struct Mol Biol. 2004; 11:812-815. [PubMed: 15332082]

Riley JL, Katz LA. Widespread distribution of extensive genome fragmentation in ciliates. Mol Biol Evol. 2001; 18:1372-1377. [PubMed: 11420375] 
Robinson T, Katz LA. Non-mendelian inheritance of two cytoskeletal genes in the ciliate Chilodonella uncinata. Mol Biol Evol. 2007; 24:2495-2503. [PubMed: 17890762]

Spring KJ, Pham S, Zufall RA. Chromosome copy number variation and control in the ciliate Chilodonella uncinata. PLoS ONE. 2013; 8(2):e56413. [PubMed: 23437129]

Sproul D, Gilbert N, Bickmore W. The role of chromatin structure in regulating the expression of clustered genes. Nat Rev Genet. 2005; 6:775-781. [PubMed: 16160692]

Struhl K. Fundamentally different logic of gene regulation in eukaryotes and prokaryotes. Cell. 1999; 98:1-4. [PubMed: 10412974]

Xu K, Doak TG, Lipps HJ, Wang JM, Swart EC, Chang WJ. Copy number variatons of 11 macronuclear chromosomes and their gene expression in Oxytricha trifallax. Gene. 2012; 505:7580. [PubMed: 22669045] 

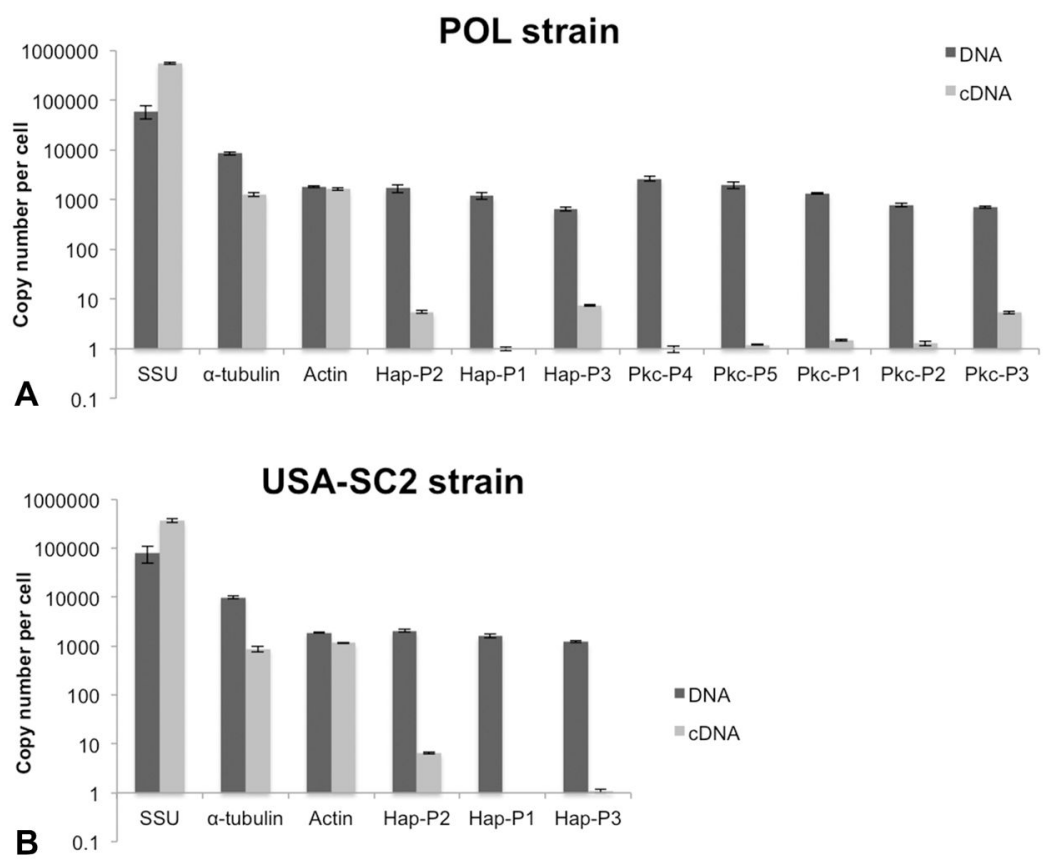

Figure 1.

Macronuclear chromosome copy numbers are not correlated with RNA levels in $C$. uncinata.

Notes: (A) 11 genes in the POL strain; (B) 6 genes in the USA-SC2 strain. Estimates for each gene family are arranged in a decreasing order of copy number. Error bars represent the standard deviation. The y-axis is a logarithmic scale. Hap: Histidine acid phosphatase family protein; $P k c$ : Protein kinase domain containing protein. 


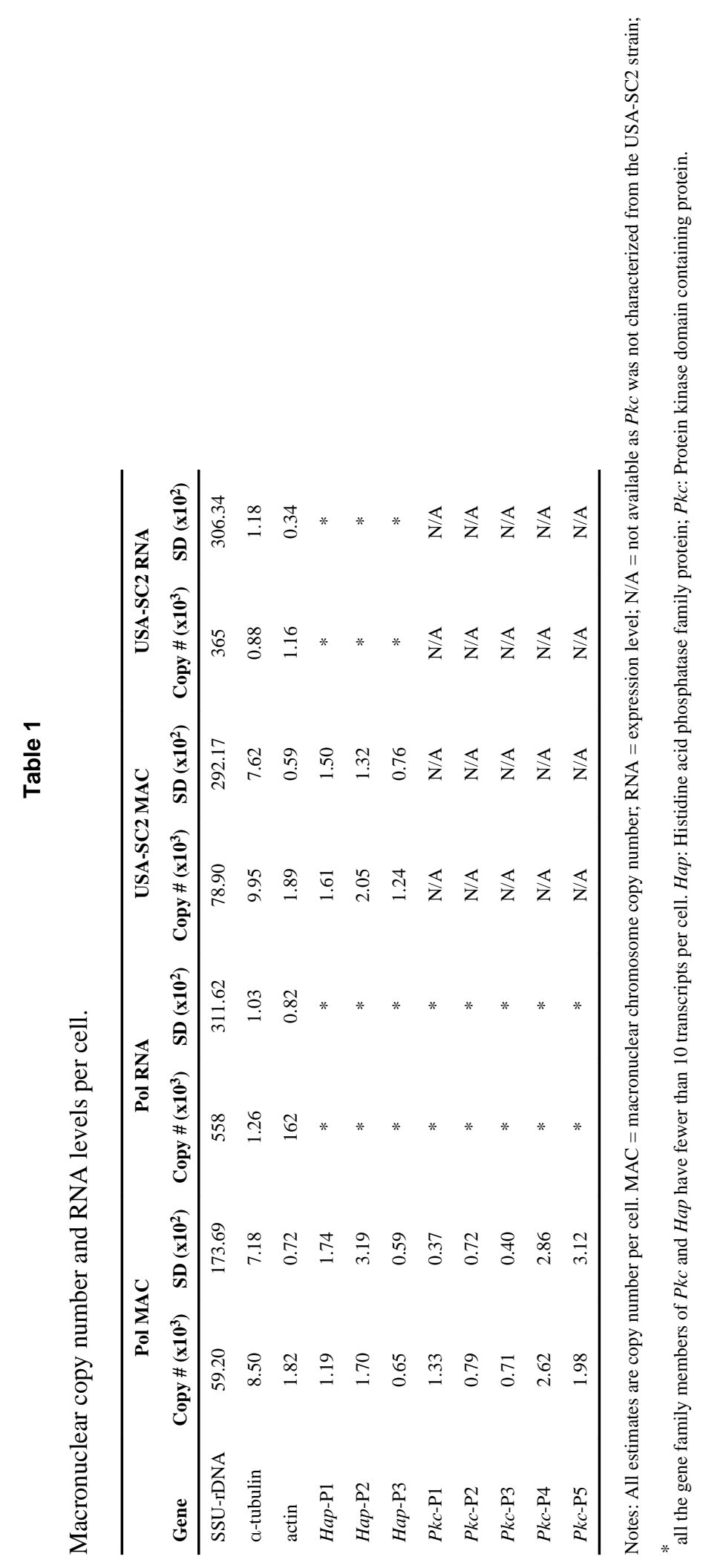

Protist. Author manuscript; available in PMC 2015 August 01. 


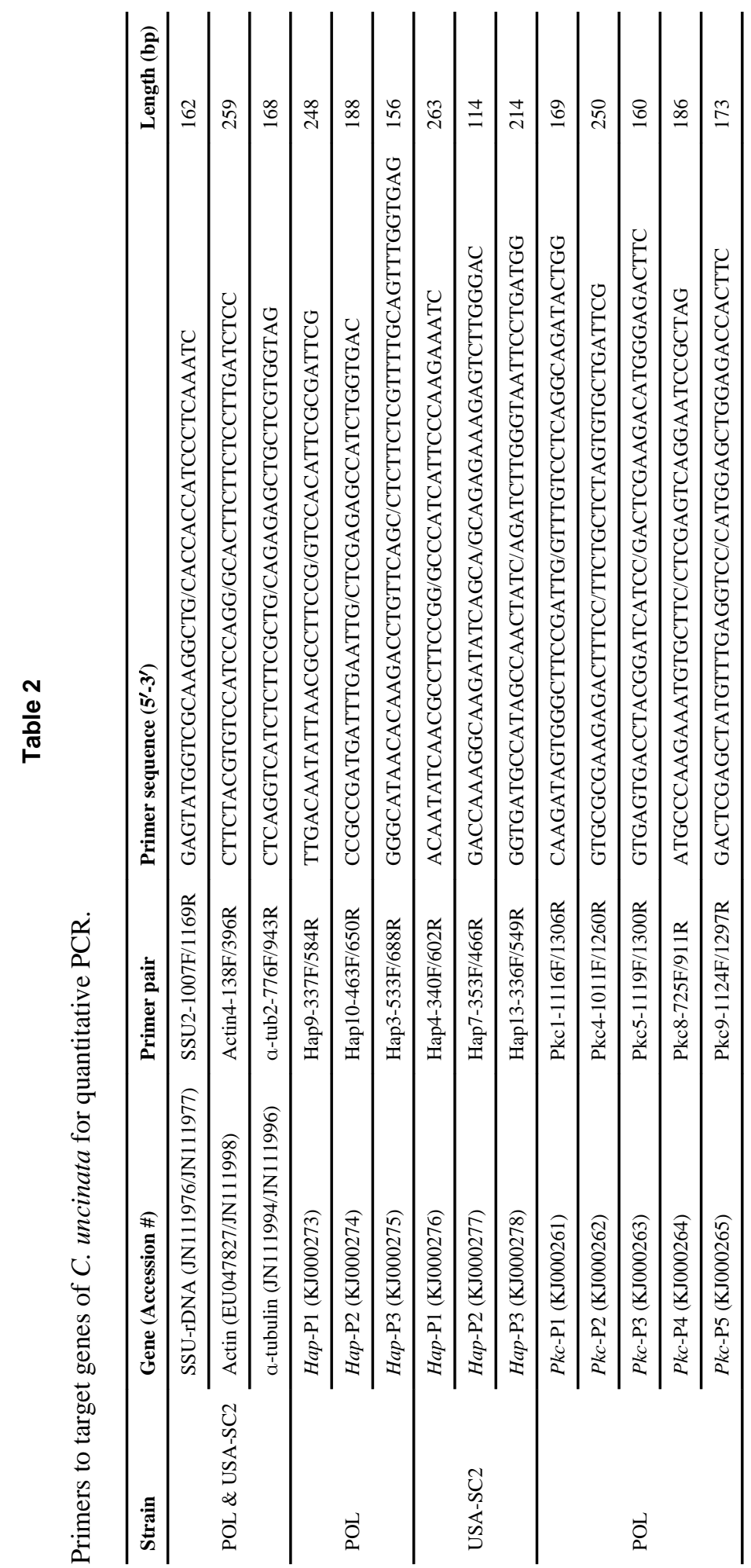

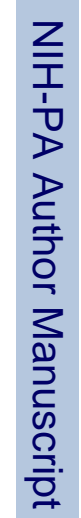

